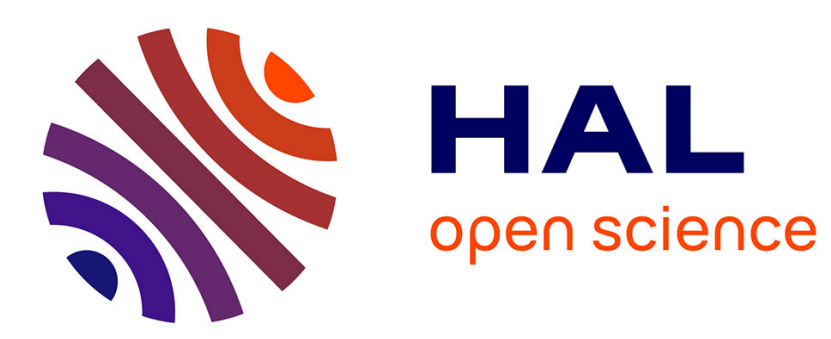

\title{
Indusion: Fusion of Multispectral and Panchromatic Images Using Induction Scaling Technique
}

\author{
Muhammad M. Khan, Jocelyn Chanussot, Laurent Condat, Annick
}

Montanvert

\section{- To cite this version:}

Muhammad M. Khan, Jocelyn Chanussot, Laurent Condat, Annick Montanvert. Indusion: Fusion of Multispectral and Panchromatic Images Using Induction Scaling Technique. IEEE Geoscience and Remote Sensing Letters, 2008, 5 (1), pp.98-102. 10.1109/LGRS.2007.909934 . hal-00348845

\section{HAL Id: hal-00348845 \\ https://hal.science/hal-00348845}

Submitted on 22 Dec 2008

HAL is a multi-disciplinary open access archive for the deposit and dissemination of scientific research documents, whether they are published or not. The documents may come from teaching and research institutions in France or abroad, or from public or private research centers.
L'archive ouverte pluridisciplinaire HAL, est destinée au dépôt et à la diffusion de documents scientifiques de niveau recherche, publiés ou non, émanant des établissements d'enseignement et de recherche français ou étrangers, des laboratoires publics ou privés. 


\title{
Indusion: Fusion of Multispectral and Panchromatic Images Using the Induction Scaling Technique
}

\author{
Muhammad Murtaza Khan, Jocelyn Chanussot, Senior Member, IEEE, Laurent Condat, and Annick Montanvert
}

\begin{abstract}
The fusion of multispectral (MS) and panchromatic (PAN) images is a useful technique for enhancing the spatial quality of low-resolution MS images. Liu recently proposed the smoothing-filter-based intensity modulation (SFIM) fusion technique. This technique upscales MS images using bicubic interpolation and introduces high-frequency information of the PAN image into the MS images. However, this fusion technique is plagued by blurred edges if the upscaled MS images are not accurately coregistered with the PAN image. In the first part of this letter, we propose the use of the Induction scaling technique instead of bicubic interpolation to obtain sharper, better correlated, and hence better coregistered upscaled images. In the second part, we propose a new fusion technique derived from induction, which is named "Indusion." In this method, the high-frequency content of the PAN image is extracted using a pair of upscaling and downscaling filters. It is then added to an upscaled MS image. Finally, a comparison of SFIM (with both bicubic interpolation and induction scaling) is presented along with the fusion results obtained by IHS, discrete wavelet transform, and the proposed Indusion techniques using Quickbird satellite images.
\end{abstract}

Index Terms-Fusion, induction, Indusion, pan sharpening, Q4 index, scaling.

\section{INTRODUCTION}

$\mathbf{T}$ HE LAUNCH of a new generation of Ikonos and Quickbird satellites has rekindled the interest of researchers in developing new fusion algorithms. Although the spatial resolution has significantly increased as compared to their predecessors, the constraint of inverse relation between spatial and spectral resolutions remains, i.e., panchromatic (PAN) images have a finer spatial resolution than multispectral (MS) images. Conversely, MS images provide a better spectral resolution.

Images with high spatial and spectral resolutions are required to improve image interpretation and pixel- or structure-wise automatic classification. High spectral resolution helps in the discrimination of land cover types, whereas high spatial resolution helps in identifying textures or determining the accurate shape and boundaries of the different objects. By making use of PAN and MS images, the fusion techniques aim at synthesizing MS images with a high spatial resolution [1].

Manuscript received January 22, 2007; revised July 12, 2007

M. M. Khan, J. Chanussot, and A. Montanvert are with the GIPSA-Lab, Grenoble Institute of Technology, 38031 Grenoble, France (e-mail: muhammad-murtaza.khan@gipsa-lab.inpg.fr; jocelyn.chanussot@gipsa-lab. inpg.fr; annick.montanvert@ gipsa-lab.inpg.fr).

L. Condat is with the Institute of Biomathematics and Biometry, National Research Center for Environment and Health (GSF), 85764 Nuremberg, Germany (e-mail: laurent.condat@gsf.de).

Color versions of one or more of the figures in this paper are available online at http://ieeexplore.iee.org.

Digital Object Identifier 10.1109/LGRS.2007.909934
Intensity-hue-saturation (IHS)-based methods are among the most popular fusion techniques for their low computational cost [2]. Together with principal component analysis based fusion, they fall in the category of component substitution methods. Although the fused MS images obtained by these methods have high spatial quality, they suffer from spectral distortions [3]. The smoothing-filter-based intensity modulation (SFIM) [4] proposed by Liu minimizes the spectral distortions while improving the spatial quality of the MS images using a simplified solar radiation and land surface reflection model. More recently, fusion methods based upon the use of discrete wavelet transform (DWT) have emerged [5], [6]. Although the images fused using DWT are not as sharp as compared to component substitution methods, they are spectrally consistent.

Generally, the fusion can be divided into two distinct parts.

1) Upscaling of the MS images, so that the upscaled MS images have the same size as the PAN image.

2) The process of fusion, which may be achieved either by the addition of the high-frequency content of the PAN image to the MS image or by substitution of the intensity image by the PAN image in case of the IHS algorithm.

As previously mentioned, the SFIM method produces spectrally consistent fused MS images. However, the problem of object blurring occurs if the low-resolution MS images, after upscaling, are not accurately coregistered with the PAN image. This problem is caused by the first part of the fusion process (upscaling), and in [4], bicubic interpolation is used for upscaling the MS images. In Section II-A, instead of using bicubic interpolation, we propose the use of the scaling technique called "Induction" for producing sharper and spectrally consistent upscaled images. In Section II-B, we propose a new fusion algorithm based upon Induction. It basically consists of extracting the high-frequency information of the PAN image and adding it to the MS images while preserving the spectral content of the MS images. In Section III, we present a comparison between different fusion techniques. Results on true Quickbird and simulated Pleiades images are presented using both visual and quantitative evaluations. Section IV presents the conclusions.

\section{Fusion Processes and Scaling Techniques}

\section{A. SFIM Using Bicubic Interpolation and Induction}

1) SFIM: This method is based upon a solar radiation and land surface reflection model. The basic idea consists of using the ratio between the high-resolution PAN image and its lowresolution version, which is obtained by low-pass filtering. Spatial details can be injected into the upscaled, coregistered, 
This article has been accepted for publication in a future issue of this journal, but has not been fully edited. Content may change prior to final publication.

low-resolution MS image without changing its spectral content as

$$
\operatorname{DN}(\lambda)_{\text {SFIM }}=\frac{\operatorname{DN}(\lambda)_{\text {low }} \mathrm{DN}(\gamma)_{\text {high }}}{\operatorname{DN}(\gamma)_{\text {mean }}}
$$

where DN stands for the digital number, and $\mathrm{DN}(\gamma)_{\mathrm{high}}$, $\operatorname{DN}(\gamma)_{\text {mean }}, \operatorname{DN}(\lambda)_{\text {low }}$, and $\operatorname{DN}(\lambda)_{\text {SFIM }}$ are the values of a pixel in the high-spatial-resolution PAN, low-spatial-resolution PAN (obtained after application of smoothing low-pass filter), upscaled low-spatial-resolution MS, and the desired highspatial-resolution MS images, respectively. The size of the smoothing filter for obtaining the $\operatorname{DN}(\gamma)_{\text {mean }}$ image is equal to the ratio of the PAN and MS image size. For Quickbird and Pleiades, the ratio is equal to 4 . Hence, the used low-pass filter is a standard $4 \times 4$ averaging filter.

The ratio between $\mathrm{DN}(\gamma)_{\text {high }}$ and $\mathrm{DN}(\gamma)_{\text {mean }}$ only retains the high-resolution edge information from the PAN image. However, if the upscaled low-resolution MS image is not accurately coregistered with the PAN image, the addition of high resolution edge information results in blurred images. In the literature, bicubic interpolation is generally used for obtaining this coregistration. We propose the use of a more accurate scaling technique called Induction [7].

2) Induction: The problem related to the upscaling of an image consists of increasing its spatial resolution. Bicubic interpolation and other linear scaling methods fail to add details and therefore introduce artifacts like blurring and ringing. On the other hand, nonlinear interpolation techniques produce visually pleasant images by estimating the localization of edge pixels with subpixel accuracy. However, there exists no definite relationship ensuring coherence between the initial and the enlarged images.

Induction [7] considers magnification as an inverse problem of reduction. This yields the condition that an enlarged image should, when reduced, give the initial image back. This condition is called the reduction constraint. If $I$ is the initial image, $a$ is the reduction ratio, $R$ is the reduction filter, and $I^{1 / a}$ is the upscaled image, then the reduction constraint can be written as $\left[I^{1 / a} * R\right] \downarrow a=I$. However, for a given image $I$ and a reduction filter $R$, there is a set of enlarged images that verifies the reduction constraint. This set of images $\left(\Omega_{I}=\{X \mid[X *\right.$ $R] \downarrow a=I\}$ ) is called the induced set. Hence, we should search the upscaled image within the induced set. Induction simply consists of projecting $J$ onto $\Omega$ so as to obtain an induced image $K$ that belongs to $\Omega$. In [7], the authors propose to upscale image $I$ and obtain the upscaled image $J$ so that the upscaled image $J$ does not satisfy the reduction constraint. For obtaining the image $J$, the authors propose to upscale the image $I$ by using Jensen's enlargement technique, which is explained in [8]. Hence, Induction uses nonlinear scaling, yet the upscaled image on reduction gives the original image back.

In [7], the authors have proposed a fast nonrecursive implementation of Induction, which is defined as

$$
K=J+[I-[J * R] \downarrow a] \uparrow a * A
$$

TABLE I

CDF 9/7 TAP FILTER COEFFICIENTS

\begin{tabular}{c|ccccc}
\hline Filter & 0 & \pm 1 & \pm 2 & \pm 3 & \pm 4 \\
\hline CDF 9 & 0.602949 & 0.266864 & -0.078223 & -0.016864 & 0.026748 \\
\hline CDF 7 & 1.115085 & 0.591271 & -0.057543 & -0.091271 & - \\
\hline
\end{tabular}

where $J$ is an upscaled image not adhering to the reduction constraint, $I$ is the initial image, $a$ is the scaling factor, $R$ and $A$ are the Cohen-Daubechies-Fauveau (CDF) 9/7 tap biorthogonal filter pair, respectively, and $K$ is the final upscaled image satisfying the reduction constraint. The interest of using the $9 / 7$ filter pair is that, being symmetric, they do not cause any shift in the filtered image, and it has been proven in [9] that their smoothness and approximation properties are better than those of the other filters. For these reasons, they have been used in the image compression standard JPEG2000. The filter coefficients used are given in Table I.

\section{B. Indusion}

In this section, a new fusion technique is developed based upon Induction. Since the process is derived from Induction and incorporates fusion, it is named "Indusion." We can rewrite (2) as

$$
K=\underline{J-[[J * R] \downarrow a] \uparrow a * A}+[I] \uparrow a * A .
$$

The underlined part of the equation represents the highfrequency information, spatial edges, while the rest represents the low-frequency part of the image. In (3), $J$ is the upscaled version of the initial image $I$. In remote-sensing image fusion, the idea is to extract the high-frequency information of the PAN image and add it to the upscaled low-resolution MS image. The above equation suggests that the high-frequency information from $J$ can be added to the upscaled version of the initial low-resolution image $I$. The idea of Indusion is to replace image $J$ by the PAN image since we want the high-frequency information of the PAN image to be added to the upscaled MS image. The modified equation becomes

$$
K=\underline{\mathrm{PAN}-[[\mathrm{PAN} * R] \downarrow a] \uparrow a * A+[I] \uparrow a * A .}
$$

The Indusion algorithm was tested on true Quickbird satellite images and simulated Pleiades images. Since the ratio between the PAN and MS images provided by them is $1: 4$, the process is divided into two stages, each with a scaling factor of 2 . The implementation of the Indusion algorithm is as follows.

1) Downscale the PAN image from 0.6- to 1.2-m resolution $\left(\mathrm{PAN}_{0.6 m} \rightarrow \mathrm{PAN}_{1.2 m}\right)$ using CDF9 filter coefficients.

2) Downscale the PAN image from 1.2- to 2.4-m resolution $\left(\mathrm{PAN}_{1.2 m} \rightarrow \mathrm{PAN}_{2.4 m}\right)$ using CDF9 filter coefficients.

Process to be repeated for each MS image:

3) Upscale the $\mathrm{MS}_{2.4 m}$ image by a factor of 2 to get $\mathrm{MS}_{1.2 m *}$ and scale it up again by a factor of 2 to get the $\mathrm{MS}_{0.6 m * \text { Temp }}$ image using CDF7 filter coefficients. 
This article has been accepted for publication in a future issue of this journal, but has not been fully edited. Content may change prior to final publication.

4) Perform histogram matching [10] between $\mathrm{PAN}_{2.4 m}$ and $\mathrm{MS}_{2.4 m}, \mathrm{PAN}_{1.2 m}$ and $\mathrm{MS}_{1.2 m *}$, and $\mathrm{PAN}_{0.6 m}$ and $\mathrm{MS}_{0.6 m * \text { Temp }}$ to get the PAN $\mathrm{PM}_{\mathrm{HM} .4 m}, \mathrm{PAN}_{\mathrm{HM} 1.2 m}$, and $\mathrm{PAN}_{\mathrm{HM}} 0.6 \mathrm{~m}$ images.

5) Upscale $\mathrm{PAN}_{\mathrm{HM} 2.4 m}$ to get $\mathrm{PAN}_{\mathrm{HM} 1.2 m *}$ using CDF7 filter coefficients.

6) Obtain the difference PANdiff HM1.2m $_{1}$ between the $\mathrm{PAN}_{\mathrm{HM} 1.2 m *}$ and PAN $\mathrm{HM}_{\mathrm{HM}} 2 m$ images.

7) Add PANdiff ${ }_{\mathrm{HM} 1.2 m}$ to the $\mathrm{MS}_{1.2 m *}$ image. This gives us $\mathrm{MS}_{\text {Fused } 1.2 m}$.

8) Upscale $\mathrm{MS}_{\text {Fused } 1.2 m}$ to get $\mathrm{MS}_{0.6 m *}$ using CDF7 filter coefficients.

9) Upscale $\mathrm{PAN}_{\mathrm{HM} 1.2 m}$ to get $\mathrm{PAN}_{\mathrm{HM} 0.6 m *}$ using CDF7 filter coefficients.

10) Obtain the difference PANdiff HM0.6m $_{\text {between the }}$ $\mathrm{PAN}_{\mathrm{HM} 0.6 m *}$ and PAN $\mathrm{HM}_{\mathrm{HM}} .6 \mathrm{~m}$ images.

11) Add PANdiff ${ }_{\mathrm{HM} 0.6 m}$ to the $\mathrm{MS}_{0.6 m *}$ image. This gives us $\mathrm{MS}_{\text {Fused0.6m }}$.

\section{EXPERIMENTAL RESULTS}

This section is divided into four subsections. In Section III-A, we present a brief overview of the index chosen to assess the quality of fused images. Section III-B presents a comparison between bicubic interpolation and Induction. Section III-C presents a comparison between the fusion results obtained by the SFIM technique using bicubic interpolation and Induction. The following subsection presents a comparison of results obtained for various fusion techniques. Before presenting the experimental results, we need to highlight the fact that we have worked on degraded spatial resolution images, i.e., PAN at 2.4-m resolution and MS at 9.6-m resolution. This was done so that the resulting fused MS images have 2.4-m resolution and can thus be compared with the original MS images. The reliability of this approach has been verified in [11].

\section{A. Quaternion Index}

Evaluating the quality of a fusion process is not a trivial task. It generally requires a number of different indices such as correlation coefficient, bias in the mean value (root-meansquare error), Spectral Angle Mapper (SAM), which quantifies spectral distortions, and Relative Dimensional Global Error in synthesis (ERGAS). Recently, Alparone et al. proposed an index, based upon quaternions, for comprehensively assessing the quality of fusion processes [12]. The proposed index is called Q4 as it can be used for the global analysis of all four MS bands of the Quickbird satellite. The index lies within the interval $[0,1]$, with 1 being the ideal value. Results are displayed as percentages of this ideal value. Using quaternion representation, the index is defined as

$$
\mathrm{Q} 4=\frac{\left|\sigma_{z 1 z 2}\right|}{\sigma_{z 1} \cdot \sigma_{z 2}} \cdot \frac{2 \sigma_{z 1} \cdot \sigma_{z 2}}{\sigma_{z 1}^{2}+\sigma_{z 2}^{2}} \cdot \frac{2 \cdot\left|\bar{z}_{1}\right| \cdot\left|\bar{z}_{2}\right|}{\left|\bar{z}_{1}\right|^{2}+\left|\bar{z}_{2}\right|^{2}}
$$

where $z_{1}=a_{1}+i b_{1}+j c_{1}+k d_{1}$, and $z_{2}=a_{2}+i b_{2}+j c_{2}+$ $k d_{2}$. This index consists of three parts. The first part consists of
TABLE II

COMPARISON OF SCALING TECHNIQUES

\begin{tabular}{c|cccc}
\hline & $\begin{array}{c}\text { Correlation CC } \\
\text { coefficients }\end{array}$ & $\begin{array}{c}\text { Contrast } \\
\text { change }\end{array}$ & $\begin{array}{c}\text { Mean } \\
\text { Bias }\end{array}$ & Q4\% \\
\hline & \multicolumn{5}{|c}{ Quickbird Mississippi } \\
\hline bicubic & 0.887 & 0.994 & 0.998 & 88.0 \\
\hline Induction & 0.893 & 0.998 & 0.998 & 89.0 \\
\hline & \multicolumn{4}{c}{ Pleiades Toulouse } \\
\hline bicubic & 0.802 & 0.955 & 0.998 & 76.5 \\
\hline Induction & 0.816 & 0.982 & 0.998 & 80.0 \\
\hline
\end{tabular}

the term $\sigma_{z 1 z 2}$, which is the hypercomplex covariance between $z 1$ and $z 2$, and $\sigma_{z 1}$ and $\sigma_{z 2}$ are standard deviations. This part of the index is sensitive to loss of correlation and spectral distortions [12]. Since the quaternions can be used for all the four bands, $a_{1}$ and $a_{2}$ represent the red, $b_{1}$ and $b_{2}$ represent the green, $c_{1}$ and $c_{2}$ represent the blue, and $d_{1}$ and $d_{2}$ represent the near-infrared (NIR) reference and fused images, respectively. Simultaneously, the second term measures contrast changes while the last term measures the mean bias of all bands. $\bar{z}_{1}$ and $\bar{z}_{2}$ are the expected values of $z 1$ and $z 2$. As the index is sensitive to spectral distortion, loss of correlation, changes in contrast, and bias, it eliminates the need for separately calculating other quality measures. Moreover, with a slight variation, the same measure can be used for a single-band evaluation (Q1, using $z_{1}=a_{1}$ and $z_{2}=a_{2}$ ) or a three-band evaluation (Q3, red, green, and blue).

\section{B. Comparison of Bicubic Interpolation and Induction (Without Fusion)}

Initially, the Q4 index was used to determine which of the two scaling techniques produces upscaled images closest to the reference MS image. The test images are comprised of portions of Mississippi and Strasbourg (taken by Quickbird) and simulated Pleiades images of Toulouse and Strasbourg. The MS images were reduced in resolution as discussed in the previous subsection and then upscaled. The comparison was made between the upscaled low-resolution MS images and the reference MS images. The results are shown in Table II.

The images scaled by Induction show an improvement of almost 3\% (76.5-80.0) for the Pleiades (Toulouse) image and $1 \%$ for the Quickbird Mississippi image. The first column that indicates the correlation coefficient demonstrates that images upscaled using Induction are more correlated to the reference image as compared to those upscaled using bicubic interpolation. Thus, we conclude that Induction is a better choice for obtaining better upscaled coregistered images than bicubic interpolation.

\section{SFIM (Using Both Bicubic Interpolation and Induction)}

The scaling process results in MS images having the same size as the PAN image. The next step consists of adding the high-frequency information of the PAN image to the MS image. This is done using the SFIM technique presented in Section II-A1. The results after fusion are shown in Table III.

The results in Table III do not show any significant difference between the two fused products. For the Toulouse images, 
This article has been accepted for publication in a future issue of this journal, but has not been fully edited. Content may change prior to final publication.

TABLE III

Q4 COMPARISON OF SCALING TECHNIQUES WiTH SFIM FUSION

\begin{tabular}{c|cccc}
\hline $\begin{array}{c}\text { Fusion } \\
\text { technique }\end{array}$ & $\begin{array}{c}\text { Correlation CC } \\
\text { coefficient }\end{array}$ & $\begin{array}{c}\text { Contrast } \\
\text { change }\end{array}$ & $\begin{array}{c}\text { Mean } \\
\text { bias }\end{array}$ & Q4\% \\
\hline SFIM & \multicolumn{5}{|c}{ Quickbird Mississippi } \\
\hline Bicubic & 0.934 & 0.999 & 0.998 & 93.2 \\
\hline Induction & 0.936 & 0.996 & 0.998 & 93.0 \\
\hline SFIM & 0.90 & Pleiades Toulouse & & \\
\hline Bicubic & 0.91 & 0.998 & 0.998 & 89.7 \\
\hline Induction & & 0.991 & 0.998 & 89.9 \\
\hline
\end{tabular}

SFIM using Induction produces better results, while for the Mississippi images, SFIM using bicubic interpolation produces better results. However, if we separately analyze the three terms of the Q4 index, one can see that the CC term in the first column is always better for the images upscaled by Induction. On the other hand, the change in contrast (second column) is greater for the Induction-scaled images than for the bicubically interpolated images. Hence, the net result is that the Q4 index for the two scaling types is approximately the same. The idea of using Induction for scaling was to get an image which is a better correlated version of the reference image as compared to the bicubically interpolated image. From the comparison of the last subsection, it is clear that the upscaled images provided by Induction are more correlated to the reference image as compared to the bicubically scaled images (Table II). However, from Table III, we conclude that better upscaling will not necessarily produce better fusion results.

\section{Comparison of Fusion Techniques}

For the purpose of comparison, the proposed Indusion technique is compared with three standard algorithms, i.e., SFIM [4], fusion using DWT [5], and IHS-based fusion [2]. The SFIM fusion technique was tested for both Induction and bicubic interpolation. The DWT algorithm tested is referred to as the ARSIS M1 method in [11]. The implemented IHS method is described in [2].

A comprehensive analysis comprises of both visual and quantitative analyses. For the quantitative analysis, the indices Q1red, Q1green, Q1blue, Q1NIR, Q3, and Q4 were calculated. Here, only the Q3 and Q4 indices are presented as they provide a global and more comprehensive idea of the quality of the fused images. However, we start with a visual analysis.

Fig. 1(c) shows the reference high-resolution MS image of Mississippi obtained by Quickbird, and all the fused images will be compared to it. Moreover, for verifying the improvement in spatial quality of the fused MS images, the highresolution PAN image and the upscaled low-resolution MS image are provided as reference in Fig. 1(a) and (b), respectively. The regions of interest are marked with red, blue, and yellow rectangles. The yellow rectangle encloses a white building. Among all the fused images, the sharpest boundaries are visible in Fig. 1(d) (Indusion) and Fig. 1(h) (IHS). However, the building appears green instead of white in Fig. 1(h) (IHS). The same problem is visible in Fig. 1(g) (DWT). The images obtained from the SFIM fusion technique are not spectrally distorted, but the building edges have diffused into the background, which causes bleeding of the edge detail. The building

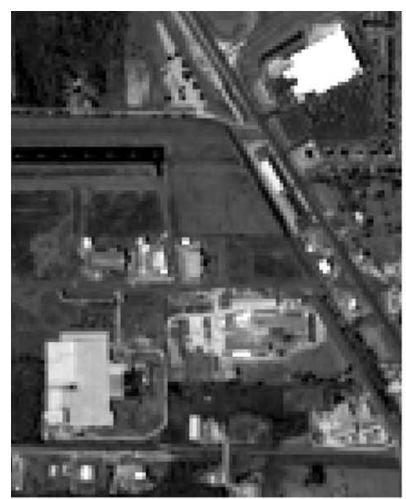

(a)

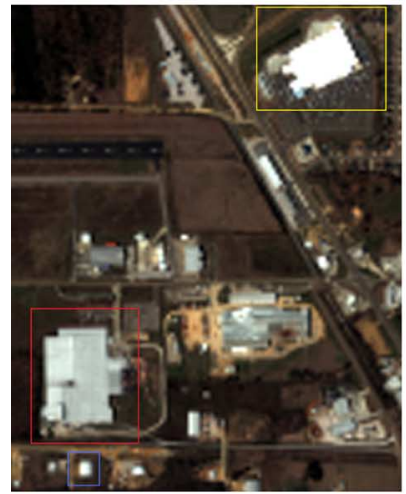

(c)

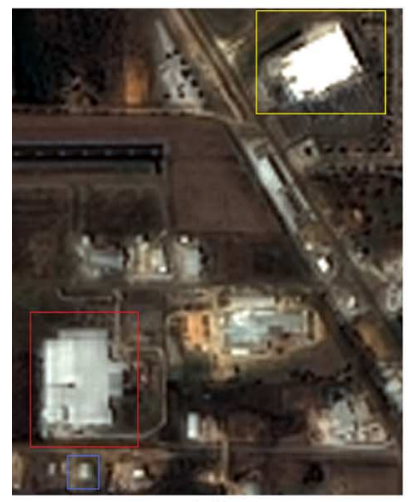

(e)

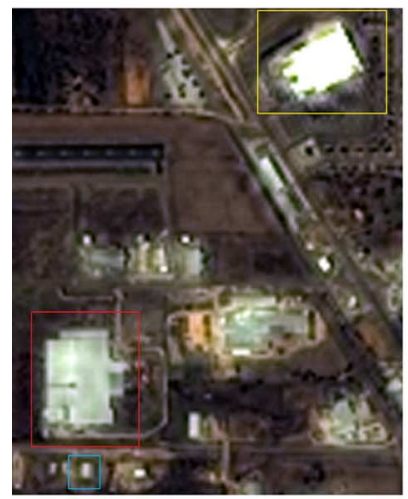

(g)

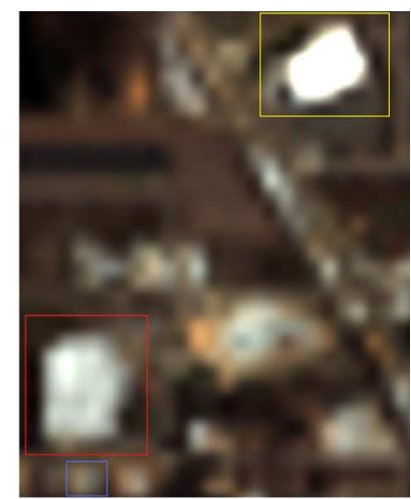

(b)

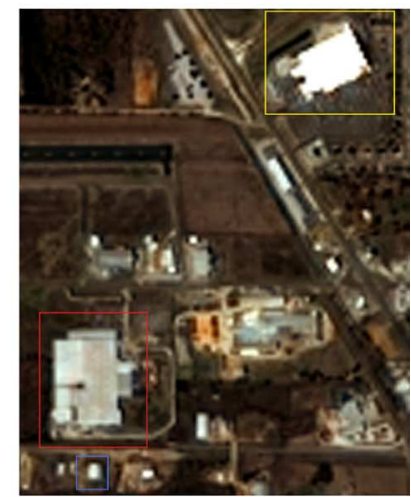

(d)

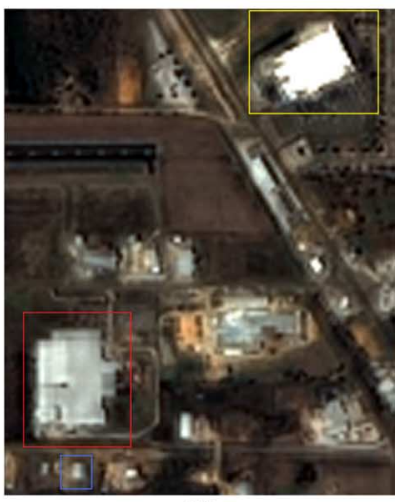

(f)

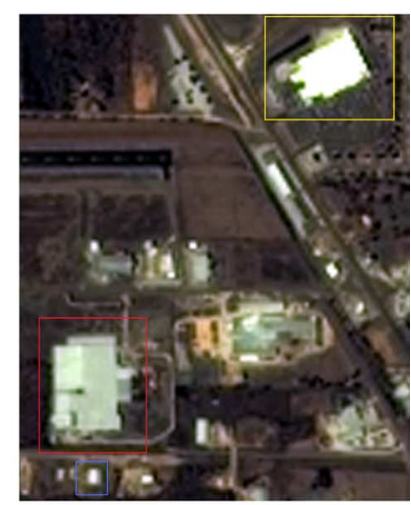

(h)
Fig. 1. Results of fusion techniques. (a) High-resolution PAN image. (b) Upscaled low-resolution MS image. (c) Reference high-resolution MS image. (d) Indusion. (e) SFIM (bicubic scaling). (f) SFIM (induction). (g) DWT. (h) IHS, wavelength PAN (445-900 nm), blue (450-520 nm), green $(520-600 \mathrm{~nm})$, and red $(630-690 \mathrm{~nm})$. 
TABLE IV

QUATERNION INDEX COMPARISON OF DIFFERENT FUSION TECHNIQUES

\begin{tabular}{c|r|lr|cr|cr|r}
\hline $\begin{array}{c}\text { Fusion } \\
\text { technique }\end{array}$ & \multicolumn{2}{c}{$\begin{array}{c}\text { Quickbird } \\
\text { Mississippi } \\
\text { Image }\end{array}$} & \multicolumn{2}{c}{$\begin{array}{c}\text { Quickbird } \\
\text { Strasbourg } \\
\text { Image }\end{array}$} & \multicolumn{2}{c}{$\begin{array}{c}\text { Pleiades } \\
\text { Toulouse } \\
\text { Image }\end{array}$} & \multicolumn{2}{c}{$\begin{array}{c}\text { Pleiades } \\
\text { Strasbourg } \\
\text { Image }\end{array}$} \\
& Q3 & Q4 & Q3 & Q4 & Q3 & Q4 & Q3 & Q4 \\
\hline SFIM-bc & 94.56 & 93.21 & 86.36 & 82.41 & 92.28 & 89.70 & 91.77 & 83.58 \\
\hline SFIM-ind & 94.38 & 93.05 & 86.58 & 82.98 & 91.89 & 89.93 & 91.60 & 84.23 \\
\hline IHS & 93.66 & 92.69 & 75.38 & 81.62 & 87.97 & 87.76 & 64.59 & 83.32 \\
\hline DWT & 92.88 & 91.58 & 84.79 & 81.64 & 89.87 & 88.32 & 89.42 & $\mathbf{8 9 . 4 9}$ \\
\hline Indusion & $\mathbf{9 4 . 5 6}$ & $\mathbf{9 3 . 5 4}$ & $\mathbf{8 8 . 1 2}$ & $\mathbf{8 4 . 3 7}$ & $\mathbf{9 4 . 6 1}$ & $\mathbf{9 0 . 9 7}$ & $\mathbf{9 5 . 4 7}$ & 87.21 \\
\hline Ind-AF & 94.05 & 93.06 & 87.83 & 83.97 & 92.62 & 89.62 & 95.12 & 87.02 \\
\hline
\end{tabular}

TABLE V

Q1 COMPARISON OF DiFFERENT Fusion TEChNiques

\begin{tabular}{c|cccc}
\hline $\begin{array}{c}\text { Fusion } \\
\text { technique }\end{array}$ & $\begin{array}{c}\text { Q1\% } \\
\text { Blue Band }\end{array}$ & $\begin{array}{c}\text { Q1\% } \\
\text { NIR Band }\end{array}$ & $\begin{array}{c}\text { Q1\% } \\
\text { Green Band }\end{array}$ & $\begin{array}{c}\text { Q1\% } \\
\text { Red Band }\end{array}$ \\
\hline & \multicolumn{4}{|c}{ Strasbourg } \\
\hline SFIM (bicubic) & 90.30 & 81.72 & 92.72 & 93.86 \\
\hline DWT & 89.42 & $\mathbf{9 0 . 4 7}$ & 90.08 & 90.70 \\
\hline Indusion & $\mathbf{9 5 . 1 8}$ & 85.66 & $\mathbf{9 6 . 1 5}$ & $\mathbf{9 6 . 0 5}$ \\
\hline
\end{tabular}

in the red rectangle has a small black square in the middle. Of all the fused images, this is clearly visible in Fig. 1(d) (Indusion). On the right of the building, there is a white rectangular object that is marked with a blue rectangle. This is clearly visible in Fig. 1(d) (Indusion). On the contrary, in all the other fused images, it either seems to be diffused with the background or appears slightly green.

As a conclusion of the visual analysis, the fused images obtained from Indusion appear to be the sharpest. Moreover, they appear to be most spectrally consistent of all the fused images. The images obtained from IHS are also sharp but are plagued by spectral distortions. The DWT algorithm has caused ringing artifacts to appear in the fused image. The images obtained from SFIM are blurred and also slightly spectrally distorted for the SFIM with Induction.

The quantitative analysis (Q4 index presented in Table IV) shows that the best fusion results are obtained by the Indusion algorithm for all the images tested except for the Pleiades Strasbourg image. The reason for this anomaly can be explained by using the Q3 and Q1 indices for this image. From the Q3 index, we see that the Indusion algorithm outperforms all the other techniques tested. This indicates that the anomaly occurs because of the fourth NIR band. Looking at Table V, we can easily conclude that Indusion outperforms the other algorithms for the red, green, and blue bands with indices of $96.05 \%$, $96.15 \%$, and $95.18 \%$, respectively. However, the results for the NIR band are best with DWT. Since the Q4 index is an average of the four bands, the DWT produces the best results as the interband variation is not significant. Otherwise, for the Q4 index, the Indusion algorithm outperforms DWT by approximately $3 \%$, IHS by $3 \%$, and SFIM (bicubic) by $1 \%$ for the Pleiades Toulouse image. For the true Quickbird image of Mississippi, the quantitative difference is not significant, but visual differences are clearly visible in Fig. 1. For the Quickbird Strasbourg image again, Indusion outperforms DWT and SFIM (bicubic). The filters mentioned in [13] were also used in the Indusion process, and the results are presented as Ind-AF in Table IV.

\section{CONCLUSION}

In this letter, the use of the Induction scaling technique has been investigated in the framework of pan sharpening. Although it provides a better upscaling than the standard bicubic interpolation, Induction in itself does not improve the fusion results of the SFIM algorithm. In the second part, a new fusion algorithm based on Induction, i.e., "Indusion," is proposed. It is tested on Quickbird and Pleiades data, and compared with three standard methods. A visual analysis and a quantitative evaluation assess the performance of the proposed algorithm. Visually, Indusion results in the sharpest and least spectrally distorted images as compared to the other techniques. Moreover, the quantitative measure, i.e., Q4 index, gives the best results for the Indusion algorithm. A tough test of spectral fidelity of the fusion process is to use a high-resolution band, which does not cover the spectral range of the low-resolution bands. Since for Quickbird images the panchromatic band does cover the spectral range of the MS images, we also tested Indusion on MODIS satellite data. For the MODIS data, the highresolution band covers wavelengths of $620-670 \mathrm{~nm}$, and the low-resolution bands cover wavelengths of 459-479, 545-565, $1628-1652$, and $2105-2155 \mathrm{~nm}$. We tested the aforementioned fusion algorithms on MODIS data, and again, Indusion outperformed other algorithms rendering spectrally consistent images with a Q4 index of $97.46 \%$ followed by DWT's index of $95.9 \%$.

\section{REFERENCES}

[1] L. Wald, Data Fusion: Definitions and Architectures_Fusion of Images of Different Spatial Resolutions. Paris, France: ENSMP, 2002.

[2] T. M. Tu, P. S. Huang, C. L. Hung, and C. P. Chang, "A fast intensity-hue-saturation fusion technique with spectral adjustment for IKONOS imagery," IEEE Geosci. Remote Sens. Lett., vol. 1, no. 4, pp. 309-312, Oct. 2004.

[3] L. Alparone, L. Wald, J. Chanussot, C. Thomas, P. Gamba, and L. M. Bruce, "Comparison of pansharpening algorithms: Outcome of the 2006 GRS-S data fusion contest," IEEE Trans. Geosci. Remote Sens., vol. 45, no. 10, pp. 3012-3021, Oct. 2007.

[4] J. G. Liu, "Smoothing filter-based intensity modulation: A spectral preserve image fusion technique for improving spatial details," Int. J. Remote Sens., vol. 21, no. 18, pp. 3461-3472, Dec. 2000.

[5] T. Ranchin and L. Wald, "Fusion of high spatial and spectral resolution images: The ARSIS concept and its implementation," Photogramm. Eng. Remote Sens., vol. 66, no. 1, pp. 49-61, Jan. 2000.

[6] J. Núnez, X. Otazu, O. Fors, A. Prades, V. Palà, and R. Arbiol, "Multiresolution-based image fusion with additive wavelet decomposition," IEEE Trans. Geosci. Remote Sens., vol. 37, no. 3, pp. 1204-1211, May 1999.

[7] L. Condat and A. Montanvert, "A framework for image magnification: Induction revisited," in Proc. ICASSP, 2005, pp. 845-848.

[8] K. Jensen and D. Anastassiou, "Subpixel edge localization and the interpolation of still images," IEEE Trans. Image Process., vol. 4, no. 3, pp. 265-295, Mar. 1995.

[9] M. Unser and T. Blu, "Mathematical properties of the JPEG2000 wavelet filters," IEEE Trans. Image Process., vol. 12, no. 9, pp. 1080-1090, Sep. 2003.

[10] D. Coltuc, P. Bolon, and J.-M. Chassery, "Exact histogram specification," IEEE Trans. Image Process., vol. 15, no. 5, pp. 1143-1152, May 2006.

[11] C. Thomas, "Fusion d'images de résolutions spatiales différentes," Ph.D. dissertation, Ecole des Mines de Paris, Sophia Antipolis, Paris, France, 2006.

[12] L. Alparone, S. Baronti, A. Garzelli, and F. Nencini, "A global quality measurement of pan-sharpened multispectral imagery," IEEE Geosci. Remote Sens. Lett., vol. 1, no. 4, pp. 313-317, Oct. 2004.

[13] B. Aiazzi, L. Alparone, S. Baronti, and A. Garzelli, "Context-driven fusion of high spatial and spectral resolution images based on oversampled multiresolution analysis," IEEE Trans. Geosci. Remote Sens., vol. 40, no. 10 , pp. $2300-2312$, Oct. 2002. 